\title{
A Rejection System for the First-Degree Formulae of some Relevant Logics
}

\author{
Ross T. BRADY \\ Philosophy Program, \\ La Trobe University \\ Ross.Brady@latrobe.edu.au \\ Received by Greg Restall \\ Published August 4, 2008 \\ http://www.philosophy .unimelb.edu.au/ajl/2008 \\ (C) 2008 Ross T. Brady
}

\section{REJECTION}

The standard Hilbert-style of axiomatic system yields the assertion of axioms and, via the use of rules, the assertion of theorems. However, there has been little work done on the corresponding axiomatic rejection of non-theorems. Such Hilbert-style rejection would be achieved by the inclusion of certain rejectionaxioms (r-axioms) and, by use of rejection-rules (r-rules), the establishment of rejection-theorems (r-theorems). We will call such a proof a rejection-proof ( $\mathrm{r}$ proof). The ideal to aim for would be for the theorems and $r$-theorems to be mutually exclusive and exhaustive. That is, if a formula $A$ is a theorem then it is not an $\mathrm{r}$-theorem, and if $A$ is a non-theorem then it is an $\mathrm{r}$-theorem. The first of these which ensures no overlap between the theorems and the r-theorems, we will call rejection-soundness (r-soundness). The second of these which ensures that all non-theorems are rejected, we will call rejection-completeness (r-completeness).

Both the set of theorems and the set of $r$-theorems are recursively enumerable, provided there are a finite number of axioms, rules, r-axioms, and r-rules. This can be ensured by use of uniform substitution rules, if necessary. If such a logic L is r-sound and r-complete then, as in [II, p. 307 (see also p. 284)], the class $T$ of all theorems of $L$ is general recursive. Then, the predicate $A \in T$ is general recursive and, by [II, p.313], L is decidable. So, there are restrictions on the logics for which both r-soundness and r-completeness hold, especially r-completeness whose proof would usually embody a decidability argument. R-soundness, on the other hand, can usually be shown either syntactically, semantically or by metacompleteness. (See Section 2 for metacompleteness.)

Though the idea of rejection goes back to Aristotle, the first rejection system was due to Łukasiewicz, who, in Chapters IV and V of [I2], set up a 
complete axiomatization of sentential logic, coupled with the axiomatic rejection of all and only the non-theorems. His positive system was as follows [I2, pp. 8O-I]:

PRIMITIVES $\sim, \supset$.

AXIOMS

I. $p \supset q \supset . q \supset r \supset . p \supset r$.

2. $\sim p \supset p \supset p$.

3. $p \supset . \sim p \supset q$.

RULES

I. $A \Rightarrow A B / p$ where $B$ is substituted for each occurrence of $p$ in $A$. (Rule of Substitution.)

2. $A, A \supset B \Rightarrow B$. (Rule of Detachment.)

For rejection, Łukasiewicz added the following: (We use the rejection sign '-’' here.)

REJECTION-AXIOM [I2, p. IO9]

I. $\dashv p$

REJECTION-RULES [I2, p.96]

I. $\vdash A \supset B, \dashv B \Rightarrow \dashv A$ (Rule of rejection by detachment.)

2. $\dashv A B / p \Rightarrow \dashv A$ (Rule of rejection by substitution.)

Łukasiewicz in [I2, pp. I09-II8], using an implicational normal form, rejected all the non-theorems of his assertion system, thus establishing $r$-soundness and r-completeness and thereby axiomatically deciding all formulae. Subsequently, Caicedo in [9] established another rejection system for sentential logic, this time without making reference to an assertion system. An induction argument on formula complexity is used to prove r-completeness.

\section{SOME PRELIMINARY CONCEPTS}

For what follows, we need to introduce the concepts of metacompleteness and degree. Meyer in [13], introduced the concept of a metacompleteness, principally for the purpose of finding an easy proof of 'If $A \vee B$ is a theorem then either $\mathrm{A}$ is a theorem or B is a theorem' for a suitable range of logics. In [13], he proved this for a wide range of quantified positive relevant logics, but it was Slaney in [15]. who managed to add negation and prove the property for the sentential relevant logics RW and TW.

To define metacompleteness for a sentential logic L, we inductively introduce the following two metavaluations, $v$ and $v^{\star}$, as in Slaney [15, pp. I62-5]. 
(i) $v(p)=F$, for all sentential variables $p$. $v^{\star}(p)=T$, for all sentential variables $p$.

(ii) $v(\mathrm{~A} \& \mathrm{~B})=\mathrm{T}$ iff $v(\mathrm{~A})=\mathrm{T}$ and $v(\mathrm{~B})=\mathrm{T}$. $v^{\star}(A \& B)=T$ iff $v^{\star}(A)=T$ and $v^{\star}(B)=T$.

(iii) $v(\mathrm{~A} \vee \mathrm{B})=\mathrm{T}$ iff $v(\mathrm{~A})=\mathrm{T}$ or $v(\mathrm{~B})=\mathrm{T}$. $v^{\star}(A \vee B)=T$ iff $v^{\star}(A)=T$ or $v^{\star}(B)=T$.

(iv) $v(\sim \mathrm{A})=\mathrm{T}$ iff $v^{\star}(\mathrm{A})=\mathrm{F}$. $v^{\star}(\sim A)=T$ iff $v(A)=F$.

(v) $v(\mathrm{~A} \rightarrow \mathrm{B})=\mathrm{T}$ iff $\vdash \mathrm{A} \rightarrow \mathrm{B}$ and, if $v(\mathrm{~A})=\mathrm{T}$ then $v(\mathrm{~B})=\mathrm{T}$, and if $v^{\star}(A)=T$ then $v^{\star}(B)=T$. $v^{\star}(\mathrm{A} \rightarrow \mathrm{B})=\mathrm{T}$.

We prove the following two lemmas.

LEMMA I For all formulae A, if $v(A)=T$ then $\vdash A$, and if $\nu^{\star}(A)=F$ then $\vdash \sim A$.

Proof: We prove these together by induction on formulae.

LEMma 2 For all formulae A, if $\vdash$ A then $v(A)=T$.

Proof: We use induction on the proof procedure for theorems.

THEOREM I L is metacomplete, i.e. $v(\mathrm{~A})=\mathrm{T}$ iff $\vdash \mathrm{A}$, for all formulae $\mathrm{A}$.

Proof: By Lemmas $\mathrm{I}$ and 2 .

The class of such metacomplete logics are called M1 logics by Slaney in [16]. Using the above metavaluations, one can also establish a number of metacompleteness properties, such as (I)-(VI) in Section 4 below. In [16), Slaney also defined M2 metacomplete logics by replacing ' $v^{\star}(A \rightarrow B)=T^{\prime}$ ' in (v) above by ' $v^{\star}(A \rightarrow B)=T$ iff, if $v(A)=T$ then $v^{\star}(B)=T$ '.

The M1 metacomplete logics include the key contraction-less logics B, DW and TW, and some weaker relevant logics such as DJ and TJ, both with conjunctive syllogism added. The contraction-less logics, EW and RW, are M2 metacomplete, as are the other contraction-less logics with the rule: $A$, $\sim B \Rightarrow \sim(A \rightarrow B)$, added. Metacomplete logics are important as they encompass contraction-less logics, which have many special properties, the logic DJ, which is conceptualized in Brady [2] and [7] as the logic of meaning containment, and TJ, which can be used to solve the set-theoretic and semantic paradoxes (see Brady [3] and [7]).

Roughly, the degree of a formula is the maximum depth of ' $\rightarrow$ ' occurring in it. Inductively:

(i) A sentential variable $p$ has degree 0 . 
(ii) If $A$ has degree $m$ and $B$ has degree $n$ then $A \& B$ and $A \vee B$ have degree $\max (m, n)$, whilst $A \rightarrow B$ has degree $\max (m, n)+1$.

Formulae of low degree are more likely to occur in practice and indeed, in Anderson and Belnap [I] and in Dunn [IO], there has been extensive study of the system $E_{\text {fde }}$ of first-degree entailments, common to relevant logics from $B$ through to R. The first-degree formulae of $\mathrm{E}$ can also be found in [I], whilst those of the weaker M1 and M2 metacomplete logics can be found in Brady [5]. It is the first degree of these M1 logics that we address in this paper.

\section{RECENT REJECTION SYSTEMS}

The issue of rejection systems has arisen in three recent contexts. The first was in connection with the metacompleteness of relevant logics including sentential constants some of which are classical. In order to fully represent their classicality, Brady in [8] considered a formal notion of non-derivability to ensure that if a sentential constant $p$ is derivable then $\sim p$ is not derivable. Thus, an axiomatic rejection system was introduced, with the following axioms and rules.

REJECTION-AXIOMS Given that, for each sentential constant $p$, none, one or both of $\vdash p$ and $\vdash \sim p$ are added, in accordance with some recursive specification, we add $\dashv p$ or $\dashv \sim p$ (or both), whenever the corresponding $\vdash p$ or $\vdash \sim p$ is not included.

REJECTION-RULES

I. $\vdash \mathrm{A} \rightarrow \mathrm{B}, \dashv \mathrm{B} \Rightarrow \dashv \mathrm{A}$.

2. $\dashv A, \dashv B \Rightarrow \dashv A \vee B$.

3. $\vdash A \vee B, \dashv A \Rightarrow \vdash B$.

Though metacompleteness and r-soundness was established for appropriate logics, the question of r-completeness was left open.

The second and related recent context is in connection with the formalization of the Law of Non-Contradiction in Brady [6]. There, it is proposed that the Law is most appropriately formalised as $\dashv A \& \sim A$, or as $\vdash A \Rightarrow \dashv \sim A$, immersed in an axiomatic rejection system.

The third is a rejection system, due to Meyer and Slaney in [I4], for Abelian $\operatorname{logic} \mathrm{A}$. They show that any formula of $\mathrm{A}$ is equivalent to a conjunctive normal form of basic intensional formulae. This theorem can then be used to establish a rejection system for $\mathrm{A}$.

\section{THE REJECTION SYSTEM $\mathrm{L}_{1 \mathrm{r}}$}

It would be nice to develop r-sound and r-complete rejection systems for a good range of decidable sentential relevent logics. To start this process, here 
we examine the first-degree metacomplete logic $\mathrm{L}_{1}$ of Brady [5]. $\mathrm{L}_{1}$ is the common first-degree fragment of a range of weak metacomplete logics which are all M1 logics. Thus, $L_{1}$ is also $B_{1}$, the first-degree fragment of the RoutleyMeyer basic logic $\mathrm{B}$, and also $\mathrm{DJ}_{1}$, the first-degree fragment of the logic $\mathrm{DJ}$ of Brady [2] and [7]. The assertion system $\mathrm{L}_{1}$ is axiomatized as follows in [5]:

AXIOMS

I. $A \rightarrow A$.

2. $A \& B \rightarrow A$.

3. $A \& B \rightarrow B$.

4. $A \rightarrow A \vee B$.

5. $B \rightarrow A \vee B$.

6. $A \&(B \vee C) \rightarrow(A \& B) \vee(A \& C)$.

7. $\sim A \rightarrow A$.

RULES

I. $\mathrm{A} \rightarrow \mathrm{B}, \mathrm{B} \rightarrow \mathrm{C} \Rightarrow \mathrm{A} \rightarrow \mathrm{C}$.

2. $A \rightarrow B, A \rightarrow C \Rightarrow A \rightarrow B \& C$.

3. $\mathrm{A} \rightarrow \mathrm{C}, \mathrm{B} \rightarrow \mathrm{C} \Rightarrow \mathrm{A} \vee \mathrm{B} \rightarrow \mathrm{C}$.

4. $\mathrm{A} \rightarrow \sim \mathrm{B} \Rightarrow \mathrm{B} \rightarrow \sim \mathrm{A}$.

NON-ENTAILMENT RULES

5. $A, B \Rightarrow A \& B$.

6. $A \Rightarrow A \vee B$.

7. $\mathrm{B} \Rightarrow \mathrm{A} \vee \mathrm{B}$.

8. $A \Rightarrow \sim \sim$.

9. $\sim A \Rightarrow \sim(A \& B)$.

Iо. $\sim B \Rightarrow \sim(A \& B)$.

II. $\sim A, \sim B \Rightarrow \sim(A \vee B)$.

As stated in [5], $\mathrm{L}_{1}$ consists of all the theorems of the first-degree entailment system $\mathrm{E}_{\mathrm{fde}}$ of Anderson and Belnap [I, p. 158], obtained by applying rules $\mathrm{I}-4$ to the axioms $\mathrm{I}-7$, with all its non-entailment theorems built up from the $\mathrm{E}_{\mathrm{fde}}$ entailments by applying rules 5-II. The following metacompleteness properties for $\mathrm{L}_{1}$ should be read in conjunction with it, most of these having allowed its axiomatization to be simplified. 
(I) If $\vdash A \vee B$ then $\vdash A$ or $\vdash B$.

(II) NOT $-\vdash \sim(A \rightarrow B)$.

(III) If $\vdash A \& B$ then $\vdash A$ and $\vdash B$.

(IV) If $\vdash \sim \sim A$ then $\vdash A$.

(V) If $\vdash \sim(A \& B)$ then $\vdash \sim A \vee \sim B$.

(VI) If $\vdash \sim(A \vee B)$ then $\vdash \sim A \& \sim B$.

Note that, by (II), the logic $\mathrm{L}_{1}$ is an M1 logic in the sense of Slaney in I6], in that it has no negated entailment theorems.

We consider the metacompleteness properties (I) and (III)-(VI) as rules for the purposes of framing the rejection-rules in what follows. We add, to the above assertion system $L_{1}$, the following rejection-axioms and rejection-rules, yielding $\mathrm{L}_{1 \mathrm{r}}$.

REJECTION-AXIOMS

I. $\dashv p \& \sim q \& r \& \sim r \rightarrow \sim p \vee q \vee s \vee \sim s$.

2. $\dashv \sim(A \rightarrow B)$.

REJECTION-RULES

I. $\vdash \mathrm{A} \rightarrow \mathrm{B}, \dashv \mathrm{A} \rightarrow \mathrm{C} \Rightarrow \dashv \mathrm{B} \rightarrow \mathrm{C}$.

2. $\vdash \mathrm{B} \rightarrow \mathrm{C}, \dashv \mathrm{A} \rightarrow \mathrm{C} \Rightarrow \dashv \mathrm{A} \rightarrow \mathrm{B}$.

3. $\dashv A, \dashv B \Rightarrow \dashv A \vee B$.

4. $\dashv A \Rightarrow \dashv A \& B$.

5. $\dashv B \Rightarrow \dashv A \& B$.

6. $\dashv A \Rightarrow \dashv \sim A$.

7. $\dashv \sim A \Rightarrow \dashv \sim(A \vee B)$.

8. $\dashv \sim B \Rightarrow \dashv \sim(A \vee B)$.

9. $\dashv \sim A, \dashv \sim B \Rightarrow \dashv \sim(A \& B)$.

Io. $\dashv A B / p \Rightarrow \dashv A$, where $B$ is uniformly substituted for $p$ in $A$.

Sentential variables are used without the use of formula schemes in the $r-$ axioms, except for RA2 which is schematic, and a rejection-substitution rule RRIo is used. Schemes are still used in the assertion system and in the statement of the r-rules. The r-rules $\mathrm{RR}_{\mathrm{I}-2}$ are reversals of rule $\mathrm{R}_{\mathrm{I}}$ of the assertion system $L_{1}$. (The reversals of rules $\mathrm{R}_{2-4}$ of $\mathrm{L}_{1}$ are not needed for the 
r-completeness argument for $\mathrm{L}_{1 \mathrm{r}}$ and so are omitted.) The r-rules $\mathrm{RR}_{3}-9 \mathrm{em}-$ brace all the requirements for the rejection-metacompleteness result of Theorem 3 to follow, some being reversals of appropriate metacompleteness properties of $\mathrm{L}_{1}$. Similar to $\mathrm{L}_{1}$ in $[5]$, we will add some $\mathrm{r}$-metacompleteness properties to round out the logic $\mathrm{L}_{1 \mathrm{r}}$.

We need the following rejection-theorems:

R-THEOREMS

I. $\dashv p .($ RAI $\times$ RRIo $)$

2. $\dashv \sim$ p. $($ RTI $\times$ RR $6 \times$ RRIo $)$

A rejection system in the style of Caicedo [9], i. e. without reference to the assertion system, can also be created, though rather tediously. This can be done by replacing the assertions $\vdash A \rightarrow B$ of $R R_{1}$ and $\vdash B \rightarrow C$ of $R_{2}$ by each of the $\mathrm{L}_{1}$ theorems actually used in the proof of Theorem 4 , i. e. in the proof of r-completeness. We then drop these theorems from the statement of the rules, yielding pure rejection rules. R-soundness and r-metacompleteness will still apply.

\section{R-SOUNDNESS AND R-METACOMPLETENESS FOR $\mathrm{L}_{1 \mathrm{r}}$}

R-soundness can be proved immediately by an easy syntactical method.

THEOREM $2 L_{1 \mathrm{r}}$ is r-sound, i.e. for all formulae A, if $\vdash$ A then NOT $-\dashv$ A.

Proof: We show that if $\dashv A$ then NOT $-\vdash A$, by induction on the r-proof procedure. The $\mathrm{r}$-axioms are non-theorems of $\mathrm{L}_{1}$. RAI is not a tautological entailment, from which it follows that it is not a theorem of any relevant logic from $\mathrm{B}$ through to $\mathrm{R}$, and $\mathrm{RA} 2$ is a metacompleteness property. The r-rules preserve non-theoremhood of $\mathrm{L}_{1}$. $\mathrm{R}_{\mathrm{I}}-2$ preserve non-theoremhood by $\mathrm{R}_{\mathrm{I}}$, $\mathrm{RR}_{3-9} 9$ by metacompleteness properties (I), (III)-(VI), and RRIo by the use of the schematic method in $\mathrm{L}_{1}$.

For rejection-metacompleteness, we inductively introduce the two rejection-metavaluations, $v_{\mathrm{r}}$ and $v_{\mathrm{r}}^{\star}$, which will relate to the rejection-theorems in a similar way to that of metavaluations and theorems of the assertion logic $\mathrm{L}_{1}$ (see Section 2).

(i) $v_{\mathrm{r}}(\mathrm{p})=\mathrm{T}$.

$$
v_{r}^{\star}(p)=F .
$$

(ii) $v_{\mathrm{r}}(\mathrm{A} \& \mathrm{~B})=\mathrm{T}$ iff $v_{\mathrm{r}}(\mathrm{A})=\mathrm{T}$ or $v_{\mathrm{r}}(\mathrm{B})=\mathrm{T}$.

$v_{\mathrm{r}}^{\star}(\mathrm{A} \& \mathrm{~B})=\mathrm{T}$ iff $v_{\mathrm{r}}^{\star}(\mathrm{A})=\mathrm{T}$ or $v_{\mathrm{r}}^{\star}(\mathrm{B})=\mathrm{T}$.

(iii) $v_{r}(A \vee B)=T$ iff $v_{r}(A)=T$ and $v_{r}(B)=T$. 
$v_{\mathrm{r}}^{\star}(\mathrm{A} \vee \mathrm{B})=\mathrm{T}$ iff $v_{\mathrm{r}}^{\star}(\mathrm{A})=\mathrm{T}$ and $v_{\mathrm{r}}^{\star}(\mathrm{B})=\mathrm{T}$.

(iv) $v_{\mathrm{r}}(\sim \mathrm{A})=\mathrm{T}$ iff $v_{\mathrm{r}}^{\star}(\mathrm{A})=\mathrm{F}$.

$v_{\mathrm{r}}^{\star}(\sim \mathrm{A})=\mathrm{T}$ iff $v_{\mathrm{r}}(\mathrm{A})=\mathrm{F}$.

(v) $v_{\mathrm{r}}(\mathrm{A} \rightarrow \mathrm{B})=\mathrm{T}$ iff $\dashv \mathrm{A} \rightarrow \mathrm{B}$.

$v_{\mathrm{r}}^{\star}(\mathrm{A} \rightarrow \mathrm{B})=\mathrm{F}$.

To establish r-metacompleteness, we prove the following lemmas.

LEMma 3 For all formulae $A$, if $\nu_{r}(A)=T$ then $\dashv A$, and if $v_{r}^{\star}(A)=F$ then $\dashv \sim A$.

Proof: We prove these together by induction on formulae.

p. By RTI and RT2, together with RRIo to introduce other variables.

A \& B. $\quad$ By RR4, RR5 and RR9.

$A \vee B . \quad \quad B y R_{3}, R_{7}$ and RR8.

$\sim$ A. $\quad$ By RR6.

$A \rightarrow B$. By RA2.

LEMma 4 For all formulae $A$, if $\dashv \mathrm{A}$ then $\nu_{\mathrm{r}}(\mathrm{A})=\mathrm{T}$.

Proof: We use induction on the proof procedure for rejection-theorems.

RAI. By (v).

RA2. By (iv), (v).

RRI-2. By (v).

RR3. By (iii).

$\mathrm{RR}_{4}$-5. By (ii).

RR6. By (iv).

RR7-8. By (iii), (iv).

RR9. By (ii), (iv).

RRıo. We use induction on formula construction to establish together (I) if $v_{r}\left(A^{B} / p\right)=T$ then $v_{r}(A)=T$, and (II) if $v_{r}^{\star}\left(A^{B} / p\right)=F$ then $v_{r}^{\star}(A)=F$. We straight-forwardly use (i)-(v) and RRIo.

THEOREM $3 L_{1 \mathrm{r}}$ is $r$-metacomplete, i.e. $v_{\mathrm{r}}(\mathrm{A})=\mathrm{T}$ iff $\dashv \mathrm{A}$, for all formulae $A$.

Proof: By Lemmas 3 and 4 .

This r-metacompleteness yields a number of properties, which enable the axiomatization of $\mathrm{L}_{1 \mathrm{r}}$ to be enhanced.

(Ir) If $\dashv A \& B$ then $\dashv A$ or $\dashv B$.

$\left(\mathrm{II}_{r}\right)$ If $\dashv A \vee B$ then $\dashv A$ and $\dashv B$. 
(III $\left.{ }_{r}\right)$ If $\dashv \sim \sim A$ then $\dashv A$.

$\left(I_{r}\right)$ If $\dashv \sim(A \& B)$ then $\dashv \sim A \vee \sim B$.

$\left(V_{r}\right)$ If $\dashv \sim(A \vee B)$ then $\dashv \sim A \& \sim B$.

These are admissible rules of $\mathrm{L}_{1 \mathrm{r}}$, in that $\mathrm{r}$-theoremhood is preserved. Thus, we can use them as such, in addition to the primitive rules RRI-IO, and, in particular, we will use $\left(\mathrm{I}_{r}\right)$ and $\left(\mathrm{II}_{r}\right)$ in determining the $\mathrm{r}$-completeness of $\mathrm{L}_{1 \mathrm{r}}$ to follow.

\section{R-COMPLETENESS FOR L 1 r}

R-completeness does involve some preliminary work. We first need to get some characterization for the non-theorems of $\mathrm{L}_{1}$. However, let us start with the theorems. For each theorem $\mathrm{A}$ of $\mathrm{L}_{1}$, using metacompleteness properties (I), (III)-(VI), as well as the rules R-7 $^{-7}$, we can establish a disjunctive normal form B consisting of a disjunction of conjunctions of basic formulae, i. e. sentential variables, negated sentential variables, entailments and negated entailments. (It is important for the negations to be pushed through first, outer ones before inner ones, as the normal-forming rules do not operate inside the scope of a negation.) Here, we include disjunctions consisting of a single disjunct, which would then be a conjunction of basic formulae. We also include conjunctions consisting of a single conjunct, which would just be a basic formula. As in Slaney [15, p. I66], by metacompleteness property (I), at least one of the disjuncts $\mathrm{C}$ of $\mathrm{B}$ must be a theorem, and hence each of its conjuncts is a theorem. Since sentential variables, negated sentential variables and negated entailments are non-theorems, the only basic formulae that can be theorems are entailments. So, the theorem disjunct $\mathrm{C}$ must consist of a conjunction of first-degree entailments. These first-degree entailments must be theorems of Anderson and Belnap's $E_{f d e}$ of [I]. Thus, for any theorem $A$ of $L_{1}$, there is a disjunctive normal form $\mathrm{B}$, consisting of a disjunction, at least one disjunct $\mathrm{C}$ of which consists of a conjunction of $\mathrm{E}_{\mathrm{fde}}$ theorems.

Non-theorems, on the other hand, are better put into conjunctive normal form. So, each non-theorem $A$ of $L_{1}$ can be put into a conjunctive normal form B, using (I), (III)-(VI), and R5-7, as above, but with a conjunction of disjunctions of basic formulae, allowing for single conjuncts and disjuncts. By $\mathrm{R}_{5}$, there is at least one conjunct $\mathrm{C}$ which is a non-theorem of $\mathrm{L}_{1}$, and, by $\mathrm{R} 6-7$, each of its disjuncts is a basic formula which is a non-theorem. Such basic formulae are sentential variables, negated sentential variables, entailments and negated entailments. Each sentential variable, negated sentential variable and negated entailment is a non-theorem, whereas the entailment non-theorems are non-theorems of $\mathrm{E}_{\mathrm{fde}}$. So, to prove $\mathrm{r}$-completeness of $\mathrm{L}_{1}$, we have to reject sentential variables, negated sentential variables and negated entailments, as well as the non-theorems of $\mathrm{E}_{\mathrm{fde}}$. Then, we have to retrace the conjunctive 
normal-forming steps, using rejection-rules and r-metacompleteness properties, until we get back to our non-theorem A. We do all this in the following theorem.

However, before we do, we first look at the structure of the non-theorems of $\mathrm{E}_{\mathrm{fde}}$. In Anderson and Belnap [I, pp. I5I-158], the theorems of $\mathrm{E}_{\mathrm{fde}}$, called tautological entailments, can all be put into normal form consisting of disjunctive normal form in the antecedent and conjunctive normal form in the consequent, i. e. $A_{1} \vee \cdots \vee A_{m} \rightarrow B_{1} \& \cdots \& B_{n}$. Whenever we pair any disjunct $A_{i}$ from the antecedent with any conjunct $B_{j}$ from the consequent, i. e. $A_{i} \rightarrow B_{j}$, we get an entailment between a conjunction of atoms and a disjunction of atoms, with at least one atom in common, where an atom is a sentential variable or a negated sentential variable. So, the non-theorems of $E_{\text {fde }}$ are those with normal forms $A_{1} \vee \cdots \vee A_{m} \rightarrow B_{1} \& \cdots \& B_{n}$, with some pair $A_{i} \rightarrow B_{j}$ having no atom from the conjuncts of $A_{i}$ in common with an atom from the disjuncts of $B_{j}$. Again, this process must be reversed for rejection.

THEOREM $4 L_{1 \mathrm{r}}$ is $r$-complete, i.e. for all formulae A, if NOT $-\vdash \mathrm{A}$ then $\dashv \mathrm{A}$.

Proof: By RTi, RT2 and RRıo, any sentential variable $\mathrm{q}$ and any negated sentential variable $\sim s$ is an $r$-theorem. By RA2, any negated entailment is an $\mathrm{r}$-theorem as RA2 is schematic. We proceed in five stages. In the first three stages, we reject the non-theorems of $\mathrm{E}_{\mathrm{fde}}$.

(I) We start with entailments between conjunctions of atoms and disjunctions of atoms. By RAI, $\dashv p \& \sim q \& r \& \sim r \rightarrow \sim p \vee q \vee s \vee \sim s$ and, by applying RRI to the antecedent and RR2 to the consequent, $\dashv p \& p \& \cdots \& p$ $\& \sim q \& \sim q \& \cdots \& \sim q \& r \& r \& \cdots \& r \& \sim r \& \sim r \& \cdots \& \sim r$ $\rightarrow \sim p \vee \sim p \vee \cdots \sim p \vee q \vee q \vee \cdots \vee q \vee s \vee s \vee \cdots \vee s \vee \sim s \vee \sim s \vee \cdots \vee \sim s$, for any number of repetitions of the variables, and with the occurrences of the variables arranged in any order and bracketed in any way within the antecedent and within the consequent. Applying RRio to expand the range of variables in their respective positions, we get $\dashv p_{1} \& p_{2} \& \cdots \& p_{i}$ $\& \sim q_{1} \& \sim q_{2} \& \cdots \& \sim q_{m} \& r_{1} \& r_{2} \& \cdots \& r_{p} \& \sim r_{1} \& \sim r_{2} \& \cdots \& \sim r_{p}$ $\rightarrow \sim p_{1} \vee \sim p_{2} \vee \cdots \sim p_{j} \vee q_{1} \vee q_{2} \vee \cdots \vee q_{n} \vee s_{1} \vee s_{2} \vee \cdots \vee s_{q} \vee \sim s_{1} \vee \sim s_{2} \vee \cdots \vee \sim s_{q}$. We can still allow repetitions of variables within each of these four categories, represented by the four types of variables and also rearrangements of variables within the antecedent and within the consequent. Note that the p's, and also the q's, can properly overlap, i. e. some p's can occur in the antecedent side and not in the consequent side or vice versa, and similarly with the q's. We confine the r's and $\sim$ 's to the same variable range on the antecedent side and the s's and $\sim$ s's to the same variable range on the consequent side. This then exhausts all possibilities for non-theorems of $\mathrm{E}_{\mathrm{fde}}$, which are entailments between conjunctions of atoms and disjunctions of atoms, provided the four distinct classes of variables, represented by the p's, q's, r's and s's are all non-empty. In order to 
reject such non-theorems of $E_{f d e}$ where at least one of these classes of variables are empty (leaving at least one of the p's, q's or r's non-empty and at least one of the p's, q's or s's non-empty), we take sub-conjunctions of the antecedent and sub-disjunctions of the consequent, this being justified by RRI and RR2. Thus, we are able to reject all entailments between conjunctions of atoms and disjunctions of atoms, where there is no atom in common.

(II) We now build up the rejection of entailments between disjunctive normal forms and conjunctive normal forms, $A_{1} \vee \cdots \vee A_{m} \rightarrow B_{1} \& \cdots \& B_{n}$, where at least one pair $A_{i} \rightarrow B_{j}$ has no atom from the conjuncts of $A_{i}$ in common with an atom from the disjuncts of $B_{j}$. These rejections of form, $\dashv A_{1} \vee \cdots \vee A_{m} \rightarrow B_{1} \& \cdots \& B_{n}$, follow from $A_{i} \rightarrow B_{j}$ by RRI and RR2, since $\vdash A_{i} \rightarrow A_{1} \vee \cdots \vee A_{m}$ and $\vdash B_{1} \& \cdots \& B_{n} \rightarrow B_{j}$, for any $i$ and $j$.

(III) All the normal-forming equivalences, $C \leftrightarrow D$, and their contextual forms, $\mathcal{C}(C) \leftrightarrow \mathcal{C}(D)$, are provable in $E_{f d e}$, and hence in $L_{1}$. So, by RRI and $\mathrm{RR}_{2}$, any non-theorem of $\mathrm{E}_{\mathrm{fde}}$ can now be rejected.

(Iv) We now reject the appropriate conjunctive normal forms, consisting of a conjunction of disjunctions of basic formulae. We have already rejected all basic formulae that are non-theorems. By repeated uses of $\mathrm{RR}_{3}$, any disjunction of rejected basic formulae is also rejected, and, by repeated $\mathrm{RR}_{4}$ and $\mathrm{RR}_{5}$, any conjunction with at least one conjunct being a rejected disjunction of basic formulae is rejected. So, we have rejected all non-theorem conjunctive normal forms of basic formulae.

(v) It remains to reject all the non-theorems of $\mathrm{L}_{1}$. We use rule versions of the normal-forming equivalences to reject arbitrary non-theorems of $\mathrm{L}_{1 \mathrm{r}}$, given the rejection of the above conjunctive normal forms. We need to use the r-rules $R_{3}-9$, together with the help of $r$-metacompleteness properties $\left(\mathrm{I}_{r}\right)$ and $\left(\mathrm{II}_{r}\right)$. We build up the inner negations first as we can only apply the rejection rules and properties $\left(\mathrm{I}_{r}\right)$ and $\left(\mathrm{II}_{r}\right)$ in conjunctive and disjunctive contexts. The use of metacompleteness properties are justified here since they essentially backtrack down a given r-proof to a certain step or steps, yielding an r-proof of this formula (or formulae) or allowing a rebuilding of the proof of a new $\mathrm{r}$-theorem from it (or them).

\section{A SIMPLE SEMANTICS FOR L 1}

One of the side benefits of an r-sound and r-complete rejection system for a logic is that one can test a potential semantics for such a logic by just proving soundness twice, once for the assertion system and once for the rejection system, both with respect to the chosen semantics. The result of this would be 
that the usual soundness would show that all theorems are valid in the semantics and that the other soundness would show that all $r$-theorems, i. e. nontheorems, are invalid in the semantics. We will appropriately call this other soundness, converse soundness or c-soundness.

One would expect this process to be easier than proving completeness, together with soundness, thus providing an opportunity to experiment with the semantics. And, as stated in Brady [5], one of the hopes in studying degree fragments of logics was to try to establish simple semantics for them. This leaves us with the immediate task of putting up a suitable semantics for the first-degree fragment $\mathrm{L}_{1}$. The semantics given in [5] is a standard RoutleyMeyer semantics over two levels, with just $T$ and $T^{\star}$ at level 1 and with the usual (possibly infinite) set of worlds at level 0 , the levels representing the maximum degree of formulae to be evaluated there.

To get some idea of how to get a simple semantics for $\mathrm{L}_{1}$, we should first look at the semantics, given in Anderson and Belnap [I, pp. 206-7], for the firstdegree fragment $\mathrm{E}_{\mathrm{fdf}}$ for the logic $\mathrm{E}$. It is a 2-stage semantics, first assessing first-degree entailments as true or false using an intensional De Morgan lattice, and then, using these assignments of truth or falsity to sentential variables, evaluating first-degree formulae in terms of these entailments and sentential variables, using classical truth-functions.

Let $\mathrm{Q}$ be a model, $\langle\mathrm{L}, v\rangle$, where $\mathrm{L}$ is an intensional De Morgan lattice and $\mathrm{v}$ is an assignment of sentential variables to elements of $\mathrm{L} . \mathrm{v}$ extends to an interpretation I for all zero-degree formulae, evaluating them as elements of $\mathrm{L}$, in the usual De Morgan manner. The valuation conditions of the semantics for $\mathrm{E}_{\mathrm{fdf}}$ are then as follows:

Let $A$ and $B$ be zero-degree formulae, positioned inside an ' $\rightarrow$ ', i. e. of depth 1 in a first-degree formula under consideration for validity. Alternatively, let $C$ and $\mathrm{D}$ be of depth 0 in such a formula.

(i) $\mathrm{I}(\mathrm{A})=\mathrm{T}$ iff $v(\mathrm{~A}) \in \mathrm{T}$, where $\mathrm{T}$ is the truth filter of $\mathrm{L}$.

(ii) $\mathrm{I}(\mathrm{A} \rightarrow \mathrm{B})=\mathrm{T}$ iff $\mathrm{I}(\mathrm{A}) \leqslant \mathrm{I}(\mathrm{B})$ in $\mathrm{L}$.

(iii) $\mathrm{I}(\sim \mathrm{C})=\mathrm{T}$ iff $\mathrm{I}(\mathrm{C})=\mathrm{F}$.

(iv) $\mathrm{I}(\mathrm{C} \& \mathrm{D})=\mathrm{T}$ iff $\mathrm{I}(\mathrm{C})=\mathrm{T}$ and $\mathrm{I}(\mathrm{D})=\mathrm{T}$.

(v) $\mathrm{I}(\mathrm{C} \vee \mathrm{D})=\mathrm{T}$ iff $\mathrm{I}(\mathrm{C})=\mathrm{T}$ or $\mathrm{I}(\mathrm{D})=\mathrm{T}$.

So, the mode of evaluation of a zero-degree subformula depends on where in the overall formula it lies.

A first-degree formula $C$ is valid in the semantics iff $I(C)=T$, for all interpretations I.

For our semantics $S_{1}$ of $L_{1}$, we should and can rectify this disparity by maintaining the same valuation conditions throughout a first-degree formula. In fact, we will project the valuation conditions for Dunn's intuitive semantics 
of $E_{\text {fde }}$ (see [IO]) over formulae of depth o as well. However, what we do is to evaluate the entailment $A \rightarrow B$ by quantifying over all interpretations, which is quite appropriate for a true entailment and it is what Dunn does for validity of $A \rightarrow B$. If we do not do this, the entailment would be evaluated like an implication, i.e. just 'if $T \in I(A)$ then $T \in I(B)$ ' in Dunn's semantics, which would not only be inappropriate for the logics $L$ of [5], of which $L_{1}$ is their first-degree fragment, but also would let in formulae such as $A \vee(A \rightarrow B)$ and $A \vee \sim B \vee(A \rightarrow B)$ as valid.

As in Dunn [IOl, valuation in $S_{1}$ is over the 4 subsets of $\{T, F\}$ and interpretation conditions are stated in terms of the membership of $\mathrm{T}$ and $\mathrm{F}$ in such subsets. The valuation $v$ assigns a subset of $\{T, F\}$ to each sentential variable and is extended to an interpretation I over all first-degree formulae, using the following conditions:

(i) $I(p)=v(p)$, for all sentential variables $p$.

(ii) $\mathrm{T} \in \mathrm{I}(\sim \mathrm{A})$ iff $\mathrm{F} \in \mathrm{I}(\mathrm{A})$.

$$
F \in I(\sim A) \text { iff } T \in I(A) .
$$

(iii) $T \in I(A \& B)$ iff $T \in I(A)$ and $T \in I(B)$.

$$
F \in I(A \& B) \text { iff } F \in I(A) \text { or } F \in I(B) .
$$

(iv) $T \in I(A \vee B)$ iff $T \in I(A)$ or $T \in I(B)$.

$$
F \in I(A \vee B) \text { iff } F \in I(A) \text { and } F \in I(B) \text {. }
$$

(v) $\mathrm{T} \in \mathrm{I}(\mathrm{A} \rightarrow \mathrm{B})$ iff, for all interpretations $\mathrm{I}^{\prime}$, if $\mathrm{T} \in \mathrm{I}^{\prime}(A)$ then $\mathrm{T} \in \mathrm{I}^{\prime}(\mathrm{B})$, and if $F \in I^{\prime}(B)$ then $F \in I^{\prime}(A)$.

$$
\mathrm{F} \notin \mathrm{I}(\mathrm{A} \rightarrow \mathrm{B}) \text {. }
$$

We add 'if $F \in I^{\prime}(B)$ then $F \in I^{\prime}(A)$ ' to the T-valuation for $A \rightarrow B$ to directly show that the contraposition rule $\mathrm{R}_{4}$ preserves truth. $\mathrm{R}_{4}$ can still be shown to hold without it, as in Dunn [ $[\mathrm{IO}$, but the duality between $T$ and $F$ here is nice. Also, the choice of F-valuation for $A \rightarrow B$ is appropriate for the logics L of [5], which are all M1 metacomplete with no negated entailment theorems (see Slaney [16).

A first-degree formula $A$ is valid in the semantics $S_{1}$ iff $T \in I(A)$, for all interpretations I. $S_{1}$ will also apply to zero-degree formulae, i. e. those without ' $\rightarrow$ ', but as with metacomplete logics there are no valid zero-degree formulae. As indicated above, if we prove soundness and c-soundness for $\mathrm{L}_{1}$ with respect to $S_{1}$ we will indeed have established soundness and completeness.

THEOREM 5 (Soundness Theorem) For all first-degree formulae A, if A is a theorem of $L_{1}$ then $A$ is valid in the semantics $S_{1}$. 
Proof: Clearly, the axioms of $\mathrm{L}_{1}$ are all valid in $\mathrm{S}_{1}$ and the rules of $\mathrm{L}_{1}$ preserve the membership of $\mathrm{T}$ in interpretations in $\mathrm{S}_{1}$.

THEOREM 6 (C-Soundness Theorem) For all first-degree formulae A, if $A$ is an $r$-theorem of $L_{1 \mathrm{r}}$ then $A$ is invalid in the semantics $S_{1}$.

Proof: We show that each axiom is invalid and that each rule preserves invalidity.

RAI. Put $v(\mathrm{p})=\mathrm{T}, v(\mathrm{q})=\mathrm{F}, v(\mathrm{r})=\{\mathrm{T}, \mathrm{F}\}$ and $v(\mathrm{~s})=\varnothing$.

RA2. By the F-valuation for $A \rightarrow B$.

RRI-2, 4-8, Io. By the corresponding validity preservation or by re-use of the interpretation yielding the invalidity of the premise to yield the invalidity of the conclusion.

$\mathrm{RR}_{3}$, 9. Because of the two premises in these rules, different interpretations can yield their respective invalidities. We need to construct an interpretation which suffices to make the conclusion invalid. For $R R_{3}$, let $T \notin I_{1}(A)$ and $\mathrm{T} \notin \mathrm{I}_{2}(\mathrm{~B})$, for some interpretations $\mathrm{I}_{1}$ and $\mathrm{I}_{2}$. We construct $\mathrm{I}_{3}$ by assigning values to the variables occurring in $A \vee B$. If the variable $p$ occurs in $A \vee B$ at depth 0 , then put $\mathrm{I}_{3}(\mathrm{p})=\mathrm{I}_{1}(\mathrm{p}) \cap \mathrm{I}_{2}(\mathrm{p})$. If $\mathrm{p}$ does not occur at depth 0 in $A \vee B$, then its value in $I_{3}$ is immaterial. By the interpretation conditions, $\mathrm{T} \notin \mathrm{I}_{1}(A)$ iff some mix of conjunctions and disjunctions of $\mathrm{T} \notin \mathrm{I}_{1}(\mathrm{p}), \mathrm{F} \notin \mathrm{I}_{1}(\mathrm{q})$, $\mathrm{T} \notin \mathrm{I}_{1}(\mathrm{~B} \rightarrow \mathrm{C})$ and $\mathrm{F} \notin \mathrm{I}_{1}(\mathrm{D} \rightarrow \mathrm{E})$, for some variables $\mathrm{p}, \mathrm{q}, \ldots$ (or none), and for some entailments $B \rightarrow C, D \rightarrow E, \ldots$ holds. These variables and entailments are all the expessions of such type that occur at depth 0 in $A$. Now, $\mathrm{I}_{3}(\mathrm{~B} \rightarrow \mathrm{C})=\mathrm{I}_{1}(\mathrm{~B} \rightarrow \mathrm{C})$ and $\mathrm{I}_{3}(\mathrm{p}) \subseteq \mathrm{I}_{1}(\mathrm{p})$, for any $\mathrm{p}$ and $\mathrm{B} \rightarrow \mathrm{C}$ at depth 0 , and so the same mix of conjunctions and disjunctions of expressions, $\mathrm{T} \notin \mathrm{I}_{3}(\mathrm{p}), \mathrm{F} \notin \mathrm{I}_{3}(\mathrm{q}), \mathrm{T} \notin \mathrm{I}_{3}(\mathrm{~B} \rightarrow \mathrm{C})$ and $\mathrm{F} \notin \mathrm{I}_{3}(\mathrm{D} \rightarrow \mathrm{E})$, holds for $\mathrm{I}_{3}$. Hence, $\mathrm{T} \notin \mathrm{I}_{3}(A)$. Similarly, by $\mathrm{I}_{3}(\mathrm{~B} \rightarrow \mathrm{C})=\mathrm{I}_{2}(\mathrm{~B} \rightarrow \mathrm{C})$ and $\mathrm{I}_{3}(\mathrm{p}) \subseteq \mathrm{I}_{2}(\mathrm{p})$, for any $\mathrm{p}$ and $\mathrm{B} \rightarrow \mathrm{C}$ at depth 0 , we also conclude $\mathrm{T} \notin \mathrm{I}_{3}(\mathrm{~B})$. Thus, $\mathrm{T} \notin \mathrm{I}_{3}(A \vee B)$, as required.

RR 9. This is similar. Given $F \notin I_{1}(A)$ and $F \notin I_{2}(B)$, for some $I_{1}$ and $I_{2}$, we construct $\mathrm{I}_{3}$ such that $\mathrm{F} \notin \mathrm{I}_{3}(A \& B)$.

\section{REFERENCES}

[i] Anderson, A. R. and Belnap, N. D., Jr., Entailment: The Logic of Relevance and Necessity, Vol. I , Princeton University Press, 1975.

[2] Brady, R. T., "Relevant Implication and the Case for a Weaker Logic", fournal of Philosophical Logic, Vol. 25 (1996), pp. 15I-I83. 
[3] Brady, R. T., "Entailment, Negation and Paradox Solution", in D. Batens, C. Mortensen, G. Priest, and J.-P. van Bendegem (eds.), Frontiers of Paraconsistent Logic, Research Studies Press, Baldock, 2000. pp. II3-I35.

[4] Brady, R. T., Editor, Relevant Logics and their Rivals, Vol. 2: A Continuation of the Work of Richard Sylvan, Robert Meyer, Val Plumwood and Ross Brady, Ashgate, Aldershot, 2003.

[5] Brady, R. T., "Degree Restrictions" in Ch. I2 of [4].

[6] Brady, R. T., "On the Formalization of the Law of Non-Contradiction", in G. Priest, J. C. Beall and B. Armour-Garb (eds.), The Law of NonContradiction: New Philosophical Essays, Oxford University Press, 2004.

[7] Brady, R. T., Universal Logic, CSLI Publications, Stanford, 2006.

[8] Brady, R. T., "Extending Metacompleteness to Classical Systems", Australasian Journal of Logic, forthcoming.

[9] Caicedo, X. "A Formal System for the Non-Theorems of the Propositional Calculus", Notre Dame fournal of Formal Logic, Vol. I9 (1978), pp. I47I5I.

[ro] Dunn, J. M., "Intuitive Semantics for First-Degree Entailment and 'Coupled Trees' ”, Philosophical Studies, Vol. 29 (1976), pp. I49-I68.

[iI] Kleene, S. C., Introduction to Meta-Mathematics, Van Nostrand, New York, 1952.

[i2] Eu kasiew icz, J., Aristotle's Syllogistic from the Standpoint of Modern Formal Logic, 2nd edn., Clarendon Press, Oxford, 1957.

[13] Meyer, R. K., "Metacompleteness", Notre Dame fournal of Formal Logic, Vol. I7 (I976), pp. 50I-516.

[I4] Meyer, R. K. and Slaney, J. K., "A, Still Adorable" in W. A. Carnielli, M. E. Coniglio and I. M. L. D'Ottaviano (eds.), Paraconsistency, The Logical Way to Inconsistency, Marcel Dekker, New York, 2002

[15] Slaney, J. K., "A Metacompleteness Theorem for Contraction-Free Relevant Logics", Studia Logica, Vol. 43 (1984), pp. 159-168.

[i6] Slaney, J. K., "Reduced Models for Relevant Logics Without WI", Notre Dame fournal of Formal Logic, Vol. 28 (1987), pp.395-407. 
The Australasian fournal of Logic (ISSN I448-5052) disseminates articles that significantly advance the study of logic, in its mathematical, philosophical or computational guises. The scope of the journal includes all areas of logic, both pure and applied to topics in philosophy, mathematics, computation, linguistics and the other sciences.

Articles appearing in the journal have been carefully and critically refereed under the responsibility of members of the Editorial Board. Only papers judged to be both significant and excellent are accepted for publication.

The journal is freely available at the journal website at

$$
\text { http://www.philosophy.unimelb.edu.au/ajl/ }
$$

All issues of the journal are archived electronically at the journal website.

SuBSCRIPTIONS Individuals may subscribe to the journal by sending an email, including a full name, an institutional affiliation and an email address to the managing editoratajl-editors@unimelb.edu.au Subscribers will receive email abstracts of accepted papers to an address of their choice. For institutional subscription, please email the managing editor atajl-editors@unimelb.edu.au

Complete published papers may be downloaded at the journal's website at http: //www.philosophy.unimelb.edu.au/ajl/ The journal currently publishes in pdf format.

Submission The journal accepts submissions of papers electronically. To submit an article for publication, send the $\mathrm{HT}_{\mathrm{E}} \mathrm{X}$ source of a submission to a member of the editorial board. For a current list of the editorial board, consult the website.

The copyright of each article remains with the author or authors of that article. 